\title{
The retinoic acid-metabolizing enzyme CYP26A1 upregulates fascin and promotes the malignant behavior of breast carcinoma cells
}

\author{
MAKOTO OSANAI and GANG-HONG LEE \\ Department of Pathology, Kochi University School of Medicine, Nankoku, Kochi 783-8505, Japan
}

Received February 6, 2015; Accepted April 6, 2015

DOI: $10.3892 /$ or.2015.4042

\begin{abstract}
The retinoic acid (RA)-metabolizing enzyme CYP26A1 has been shown to efficiently enhance the oncogenic potential of breast cancer, suggesting a potential oncogenic function. We previously demonstrated that CYP26A1 confers unique cell survival properties by modulating the expression of a variety of genes and identified a number of genes that drive the cells into the oncogenic state. Accumulating evidence suggested that fascin is overexpressed in various types of cancer, primarily leading to increased cell motility. Therefore, in the present study, we examined fascin, an actinbundling protein, using immunohistochemical and SA- $\beta$-gal staining as well as TUNEL and colony forming assays. The results of the present study showed that the expression levels of fascin increased significantly in response to CYP26A1 overexpression and, conversely, treatment with all-trans RA downregulated the expression of fascin. In addition, primary breast carcinoma samples, particularly hormone receptornegative carcinomas and CYP26A1-overexpressing cancers, expressed elevated levels of fascin. Notably, fascin contributed to the ability of breast carcinoma cells to escape premature senescence and exhibit enhanced cell apoptotic resistance, promoting anchorage-independent growth properties. Fascin also promoted cell motility and the invasiveness of CYP26A1expressing breast carcinoma cells. These data suggest that fascin expression is modulated by the intracellular RA status regulated by the expression of CYP26A1 and plays a significant role in the malignant behavior of CYP26A1-expressing breast carcinoma cells. CYP26A1 exerts oncogenic functions during breast carcinogenesis. Therefore, CYP26A1-mediated oncogenic characteristics may be partially responsible for the elevated expression of fascin.
\end{abstract}

Correspondence to: Dr Makoto Osanai, Department of Pathology, Kochi University School of Medicine, Kohasu, Oko-cho, Nankoku, Kochi 783-8505, Japan

E-mail: osanaim@kochi-u.ac.jp

Key words: retinoic acid, retinoic acid-metabolizing enzyme CYP26A1, oncogene, fascin, breast cancer

\section{Introduction}

Fascin-1 (FSCN1, also known as fascin) is a key actin-bundling protein that organizes F-actin into well-ordered, tightly packed, parallel bundles in cells (1-3). Fascin is widely expressed in mesenchymal tissues and cells in the nervous system. However, regulation of the fascin-actin interaction is complex and involves components of the extracellular matrix, peptide factors and other actin-binding proteins. Accumulating evidence suggests that the expression of fascin is increased in various types of cancer, including breast, lung, liver and colon $(4,5)$. Notably, cancer cells that express high levels of fascin exhibit aggressive characteristics including a high migratory phenotype and invasiveness, suggesting a positive correlation between fascin overexpression and the aggressive behavior of cancer cells (4-9).

Retinoic acid (RA) is a critical regulator of differentiation, proliferation and apoptosis in various cell types. The RA-metabolizing enzyme CYP26A1 has been shown to promote cell survival and contribute to the oncogenic potential of breast carcinoma cells, suggesting that this protein has an oncogenic function (10). Consistent with this finding, enhanced RA metabolism has been observed in various types of cancer and elevated levels of CYP26A1 have been reported in a number of cancer cell types (11-13). The above-mentioned studies suggested that CYP26A1 confers unique cell-survival properties on cells and modulates the expression of a variety of genes to favor cell survival. We performed a series of preliminary experiments using high-resolution oligonucleotide-based microarray analyses on CYP26A1-overexpressing breast carcinoma cells. These studies identified a number of genes that drive the cells into the oncogenic state. In the present study, we focused on fascin as a potential downstream target of CYP26A1. Fascin primarily promotes cell motility and invasiveness; therefore, its potential role in CYP26A1-mediated cancer aggressiveness is consistent with previous findings demonstrating that the enhanced expression of CYP26A1 is associated with increased cell motility and invasiveness (10).

The relevance of increased fascin activity in human breast cancer remains unclear. In the present study, we demonstrated that fascin expression increases significantly in response to constitutive CYP26A1 overexpression. Small-interfering RNA (siRNA)-mediated suppression of fascin inhibited the malignant behavior of CYP26A1-expressing breast carcinoma 
cells. The results suggested that CYP26A1-mediated malignant behavior may be partially, albeit significantly responsible for the elevated expression of fascin.

\section{Materials and methods}

Cell culture and transfection. The MCF-7, MDA-MB-231, ZR75-1 and T47-D breast cancer cell lines were obtained from a local distributor (Summit Pharmaceuticals International, Tokyo,Japan) of the American Type Culture Collection (ATCC; Manassas, VA, USA). The AC2M2 breast cancer cell line was generously provided by Dr Bruce Elliott (Cancer Research Institute, Queen's University, Kingston, ON, Canada) (14). The cells were maintained in Dulbecco's modified Eagle's medium (DMEM; Sigma, St. Louis, MO, USA) supplemented with 10\% fetal bovine serum (FBS; Invitrogen, Carlsbad, CA, USA), $100 \mathrm{U} / \mathrm{ml}$ penicillin and $100 \mu \mathrm{g} / \mathrm{ml}$ streptomycin (Sigma).

The cells were transfected with the pcDNA3.1(-) expression vector (Invitrogen) containing the full-length CYP26A1 cDNA and a vector encoding fascin-specific or control siRNA (both from OriGene, Rockvsille, MD, USA) using FuGENE ${ }^{\circledR} 6$ (Roche, Basel, Switzerland). Transfected clones were selected using $0.8-\mathrm{mg} / \mathrm{ml} \mathrm{G} 418$ for CYP26A1 and $1.2-\mu \mathrm{g} / \mathrm{ml}$ puromycin for fascin. After $>14$ days of selection, drug-resistant clones were selected and screened for CYP26A1 and fascin expression. Preliminary experiments revealed that the phenotypes of at least three independent clones of transfectants were similar, but not identical. Therefore, cell populations were mixed to establish stable transfected cell lines, avoid possible clonal variation and exclude the possibility that the cloning procedures were selected for a specific phenotype. Empty vector-transfected cells were used as controls.

Reverse transcription-quantitative polymerase chain reaction $(R T-q P C R)$ analysis. Total RNA was extracted using a TRIzol ${ }^{\circledR}$ reagent and RT-PCR was performed using an RT-PCR kit (Superscript II) (both from Invitrogen). Samples were incubated at $42^{\circ} \mathrm{C}$ for $50 \mathrm{~min}$ and the reactions were terminated by incubation at $70^{\circ} \mathrm{C}$ for $15 \mathrm{~min}$, according to the manufacturer's instructions. The cDNA was incubated with 0.5 units of Taq DNA polymerase (Takara, Shiga, Japan) to amplify the human CYP26A1, fascin and GAPDH genes. The cycling conditions used were: $20-40$ cycles of $30 \mathrm{sec}$ at $96^{\circ} \mathrm{C}, 30 \mathrm{sec}$ at $58^{\circ} \mathrm{C}$ and $1 \mathrm{~min}$ at $72^{\circ} \mathrm{C}$, followed by a final elongation time of $7 \mathrm{~min}$ at $72^{\circ} \mathrm{C}$. The PCR primers used were: CYP26A1, 5'-GCCTCT CTAACCTGCACGAC-3' and 5'-GCTCTTCTCGCACTT TCTGG-3'; fascin, 5'-AGGACGAGCTCTTTGCTCTG-3' and 5'-TGCCTGTGGAGTCTTTGATG-3'; and GAPDH, 5'-ACC ACAGTCCATGCCATCAC-3' and 5'-TCCACCACCCTG TTGCTGTA-3'. The expression of each gene of interest was analyzed using cycling parameters that had been optimized previously to produce expression linearity, allowing the semiquantitative analysis of signal intensity. PCR reactions were repeated in three independent experiments to ensure that the quantified expression was reproducible. Densitometric analysis of bands on the agarose gels was performed using ImageJ software (National Institutes of Health, Bethesda, MD, USA).

Western blotting. Aliquots of whole cell lysates $(20 \mu \mathrm{g})$ were separated on $12 \%$ sodium dodecylsulfate(SDS)-polyacrylamide gels and electroblotted onto nitrocellulose membranes. The membranes were then immunoblotted with antibodies against fascin (Dako, Glostrup, Denmark), CYP26A1, p16 ${ }^{\mathrm{INK} 4 \mathrm{~A}}$, p21 $1^{\text {Waf1/Cip1 }}$, p27 $7^{\text {Kipl }}$ (all from Santa Cruz Biotechnology, Inc., Santa Cruz, CA, USA), p53 (Novocastra, Newcastle, UK), membrane type-1 matrix metalloproteinase (MMP-14; Abcam, Cambridge, UK) and $\beta$-actin (Sigma). The membranes were incubated with the appropriate peroxidase-labeled secondary antibodies (Dako) and bands were visualized using enhanced chemiluminescence (GE Healthcare, Buckingham, UK).

Immunohistochemistry. The breast cancer tissue microarray slide (SuperBioChips Laboratories, Seoul, Korea) was deparaffinized, and antigen retrieval was performed by microwave heating $(500 \mathrm{~W})$ in citrate buffer for $15 \mathrm{~min}$. The slides were then incubated overnight at $4{ }^{\circ} \mathrm{C}$ with monoclonal primary antibodies against fascin (1:100 dilution, clone no. 55K-2; Dako) or CYP26A1 (1:50 dilution, clone no. F27 P6 A1; Santa Cruz Biotechnology, Inc.). The sections were then incubated with EnVision $^{\mathrm{TM}}$ (Dako) for $30 \mathrm{~min}$ at room temperature. Positive staining was graded according to the percentage of the stained tumor cells: $3+$ (strong), staining in $>50 \%$ of cells; $2+$ (moderate), staining in $25-49 \%$ of cells, $1+$ (weak), staining in $5-24 \%$ of cells, 0 (negative), no or faint staining in $<5 \%$ of the cells. Fascin expression was considered positive with staining grades of $2+$ or $3+$ and negative with 0 or $1+$. The data were confirmed in independent duplicate analyses. The original immunohistochemical findings of the microarray array analysis are available on request.

Senescence-associated $\beta$-galactosidase (SA- $\beta$-gal) staining. The MCF-7 cells were exposed to mild oxidative stress by incubation with $10 \mu \mathrm{M} \mathrm{H}_{2} \mathrm{O}_{2}$ for up to 6 days in the presence or absence of $1 \mu \mathrm{M}$ all-trans retinoic acid (atRA; Sigma) and senescent cells were visualized using SA- $\beta$-gal staining (15).

Cell cycle analysis by flow cytometry. The MCF-7 cells were subjected to mild oxidative stress with $10 \mu \mathrm{M} \mathrm{H}_{2} \mathrm{O}_{2}$ for 6 days prior to being harvested by centrifugation and permeabilized with $70 \%$ ethanol for $10 \mathrm{~min}$. After being washed with phosphate-buffered saline (PBS), the cells were treated with PBS containing $100 \mu \mathrm{g} / \mathrm{ml}$ DNase free-RNase A (Invitrogen) at $37^{\circ} \mathrm{C}$ for $30 \mathrm{~min}$. The cells were then suspended in PBS containing $40 \mu \mathrm{g} / \mathrm{ml}$ propidium iodide solution for $15 \mathrm{~min}$. The DNA content was analyzed using FACSCalibur ${ }^{\mathrm{TM}}$ (BectonDickinson, Franklin Lakes, NJ, USA).

Apoptosis induction. Oxidative stress-induced apoptosis was stimulated by incubating cells in $0-100 \mu \mathrm{M} \mathrm{H}_{2} \mathrm{O}_{2}$ for $24 \mathrm{~h}$. Anoikis (anchorage-dependent apoptosis) was then induced to prevent the cells from adhering to the culture dishes in the presence or absence of $1 \mu \mathrm{M}$ atRA. In some experiments, inhibitors of mitogen-activated protein kinase (MAPK; PD98059, $50 \mu \mathrm{M}$ ), p38 MAPK (SB203580, $20 \mu \mathrm{M}$ ) or phosphoinositide-3-kinase (PI3K; LY294002, $20 \mu \mathrm{M}$ ) were added (all from Sigma).

Terminal deoxynucleotidyl transferase-mediated nick end-labeling (TUNEL) and cell proliferation assays. Apoptosis was assessed in the MCF-7 cells cultured on collagen-coated 
glass coverslips using a TUNEL assay. Apoptotic cells were visualized using an In Situ Cell Death Detection kit (Roche). The procedure was also performed without terminal deoxynucleotidyl transferase as a negative control. To examine the cell proliferation rate, the cells were manually counted every $24 \mathrm{~h}$ up to day 6 after an equal number of cells were plated. In addition, DNA synthesis in the cells was assessed using Ki-67 labeling. For immunohistochemistry, the cells on a coverslip were incubated with a primary antibody against Ki-67 and then incubated with EnVision ${ }^{\mathrm{TM}}$ (both from Dako) for 30 min at room temperature. The results were confirmed in triplicate independent analyses.

Colony forming assays in two- (2-D) and three-dimensional (3-D) cultures. The MCF-7 cells $\left(5 \times 10^{5}\right.$ cells/well) were plated in 6-well plates $24 \mathrm{~h}$ prior to exposure to a lethal dose of oxidative stress $\left(500 \mu \mathrm{M} \mathrm{H}_{2} \mathrm{O}_{2}\right)$ for $24 \mathrm{~h}$. The small number of cells that survived was then cultured for $>7$ days to form individual colonies. Soft agar assays were performed in $6-\mathrm{cm}$ dishes to assess colony formation in three dimensions. Breast carcinoma cells $\left(2.5 \times 10^{3}\right)$ were uniformly suspended in $6 \mathrm{ml}$ of $0.33 \%$ agarose gel with DMEM supplemented with 5\% FBS, and then overlaid onto a base layer of $1 \%$ agarose gel. The plates were incubated for at least 3 weeks and cell clusters $>50 \mu \mathrm{m}$ in diameter were defined as positive.

Invasion assay. A cell suspension $\left(5 \times 10^{5}\right.$ cells of MDA-MB-231 in $0.5 \mathrm{ml}$ of DMEM with $0.5 \%$ FBS) was added to 24 -well cell culture inserts with $8-\mu \mathrm{m}$ pores that had been pre-coated with Matrigel $^{\mathrm{TM}}$ matrix (both from Becton-Dickinson). DMEM containing $10 \%$ FBS was added to the lower chamber to create a chemotactic gradient. The number of the invasive cells was then estimated after $48 \mathrm{~h}$ of incubation. The lower surface of the upper chamber was wiped with a cotton swab and any cells that passed through the filters onto the lower surface of the cell culture inserts were quantified.

Gelatin zymography. The gelatinolytic activities of MMPs were determined in MDA-MB-231 cell supernatants. Aliquots of conditioned media were separated by electrophoresis under non-reducing conditions without heating in $0.1 \%$ gelatin-containing $9 \%$ polyacrylamide gels. The gels were washed with $2.5 \%$ Triton $^{\mathrm{TM}} \mathrm{X}-100$ for $1 \mathrm{~h}$ to remove the SDS, and were then incubated overnight at $37^{\circ} \mathrm{C}$ in a Tris-based buffer. The gels were then stained for $30 \mathrm{~min}$ with $0.5 \%$ Coomassie brilliant blue. Clear bands appeared on the blue background in the areas with gelatinolytic activity.

Wound-healing assay. The MDA-MB-231 cells were grown to confluence and a scratch was made through the cell monolayer using a pipette tip. After being washed twice with PBS, fresh media were added and the wounded monolayer was photographed overtime.

Statistical analysis. The cells or colonies of interest for each assay were counted under low magnification (x100) in 10 randomly selected fields using light microscopy. Data are presented as means \pm standard deviation (SD) from at least three independent experiments, each performed in triplicate wells. Statistical differences were analyzed using the Student's t-tests and data were considered to indicate a statistically significant result when $\mathrm{P}<0.05$.

\section{Results}

Upregulation of fascin in response to CYP26A1 overexpression. We performed a series of preliminary experiments on CYP26A1-overexpressing breast carcinoma cells, and identified a number of genes that drive the cells into the oncogenic state. The results confirmed that the CYP26A1-expressing cells expressed elevated levels of fascin. As expected, fascin was constitutively expressed in the MCF-7 cells and its expression was elevated in CYP26A1-transfected cells (Fig. 1A). The fascin expression was also significantly upregulated in other CYP26A1-overexpressing breast carcinoma cells (Fig. 1B), suggesting that its effect on fascin occurred in a number of cell lines. In addition, treatment with atRA downregulated the expression of fascin in a dose- and timedependent manner (Fig. 1C). Conversely, fascin had no effect on the CYP26A1 expression, whereas the siRNA-mediated knockdown of CYP26A1 downregulated the expression of fascin (Fig. 1D). These results suggested that fascin expression was modulated by the intracellular RA levels, regulated by the expression of CYP26A1.

Fascin overexpression in primary breast carcinomas. The expression of fascin in breast carcinomas was also investigated using tissue microarray. Although no positive reactivity was detected in the ducts and lobules of normal breast tissues, strong cytoplasmic staining for fascin was observed in 19 of $50(38 \%)$ breast carcinoma samples (Fig. 2A). In addition, metastatic carcinomas had a significantly higher expression of fascin, when compared with non-metastatic primary carcinomas (Fig. 2B), suggesting a potential role for fascin in tumor progression. Notably, fascin was upregulated in hormone receptor-negative cancers (Fig. 2C). Eighteen of 33 (55\%) tumors that were negative for the estrogen receptor (ER) and progesterone receptor $(\mathrm{PR})$ were fascin-positive, but fascin was negative $(0 \%)$ in all tumors that were ER- and PR-positive. A positive correlation between fascin and CYP26A1 expression was observed in 12 of 22 (55\%) CYP26A1-positive tumors, whereas only 6 of 37 (16\%) CYP26A1-negative tumors were stained positive for fascin $(\mathrm{P}=0.0132$; Fig. $2 \mathrm{D}$ and $\mathrm{E})$. These data provide further evidence of a correlation between CYP26A1 and fascin. Although a larger cohort study of breast cancers showed that the CYP26A1 expression was significantly associated with patient survival (16), fascin expression did not correlate with the patient age, gender, tumor histology, the pTNM stage, the primary tumor status, the number of lymph nodes involved or the p53 and HER2 expression levels.

Suppression of fascin induces premature senescence. The effect of fascin on cell senescence was assessed since premature senescence is an intrinsic fail-safe mechanism against carcinogenic insults $(15,17)$. Senescence was assessed by measuring SA- $\beta$-gal enzymatic activity. Since a high level of oxidative stress efficiently induced apoptosis, we examined the level of oxidative stress required to induce senescence in the cells, but not to undergo apoptosis in our preliminary study. When the MCF-7 cells were exposed to mild oxidative stress that was 
A
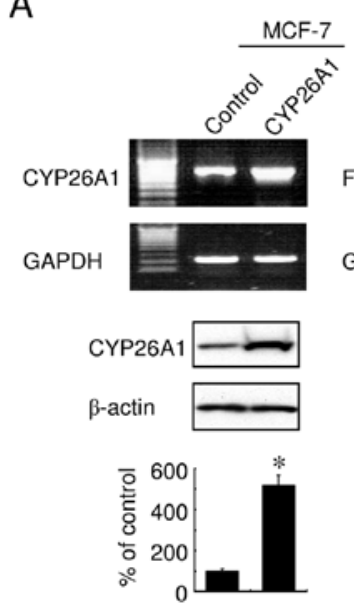

C

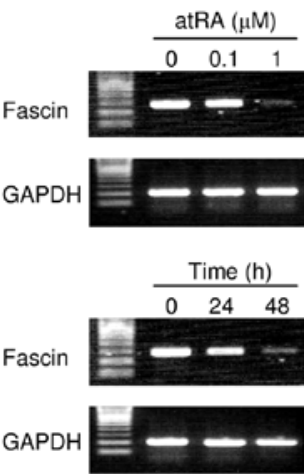

B
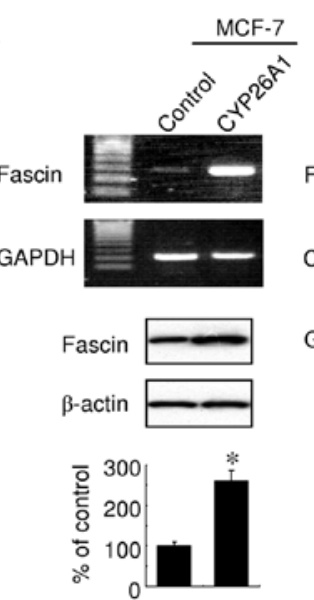

Fascin

CYP26A1

GAPDH

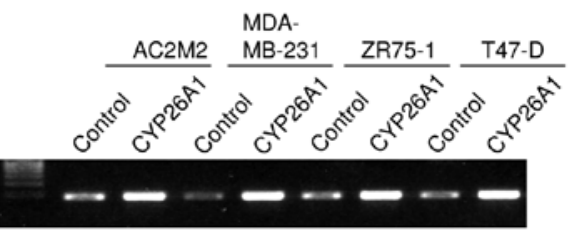

1
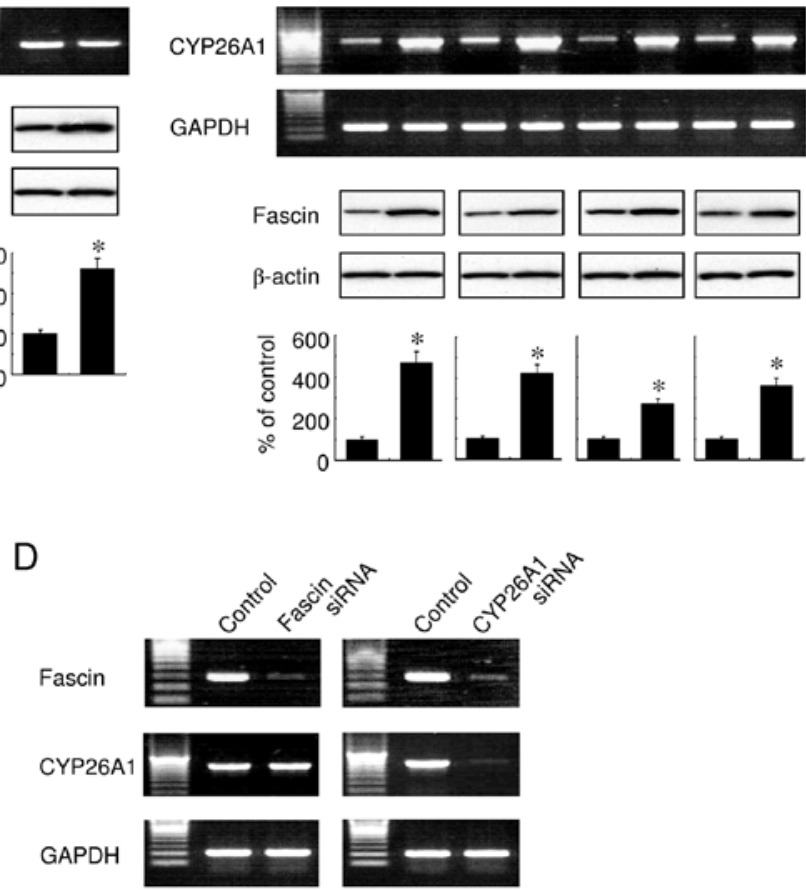

Figure 1. Enhanced expression of fascin in CYP26A1-overexpressing breast carcinoma cells. (A and B) Fascin and CYP26A1 expression was analyzed in (A) MCF-7 and (B, as indicated) other cells with or without CYP26A1 overexpression and the expression was assessed by RT-qPCR (upper panels). The expression level of these proteins was also examined by western blotting and its quantitative analyses (lower panel). (C) Treatment with atRA suppresses fascin expression in a dose- (upper panel; $0,0.1$ and $1 \mu \mathrm{M}$ atRA for $48 \mathrm{~h}$ ) and time- (lower panel; $1 \mu \mathrm{M}$ atRA for 0,24 and $48 \mathrm{~h}$ ) dependent manner in MCF-7 cells, as assessed by RT-qPCR. (D) Transfection of fascin-specific siRNA suppresses the expression of fascin, but not CYP26A1 in the MCF-7 cells. However, the siRNA-mediated suppression of the CYP26A1 downregulated fascin expression. $\mathrm{P}<0.05$ vs. the control cells $\left(^{*}\right)$. atRA, all-trans retinoic acid.

insufficient to induce apoptosis, knocking down fascin caused prominent phenotypic alterations (Fig. 3A), including stimulating an enlarged, flat morphology with multiple vacuoles and cytoplasmic protrusions. In addition, the SA- $\beta$-gal activity was significantly increased after transfection with fascin-specific siRNA, in the presence and absence of atRA (Fig. 3B). These results suggested that atRA and fascin suppression synergistically enhanced cell senescence, since atRA inhibited the fascin expression (Fig. 1C). Although the number of senescent cells markedly decreased when CYP26A1 was overexpressed, fascin suppression significantly increased the number of the SA- $\beta$-gal-positive cells (Fig. 3B). These results suggested that elevated levels of fascin play a role in the signaling pathways that mediate the escape from premature senescence.

Downregulation of fascin induces growth arrest during oxidative stress. To investigate the effect of fascin signaling on MCF-7 cell proliferation, the cell number was analyzed in a time-dependent manner. Manual cell counting revealed no significant differences in the growth and DNA synthesis rates, regardless of the expression of fascin. However, cells transfected with fascin-specific siRNA that were exposed to mild oxidative stress exhibited a significantly decreased cell growth and DNA synthesis rate as assessed using Ki-67 labeling (Fig. 3C).
Consistent with this, the expression of cyclin-dependent kinase $(\mathrm{CDK})$ inhibitors, including $\mathrm{p} 16^{\mathrm{INK} 4 \mathrm{~A}}, \mathrm{p} 21^{\mathrm{Waf} 1 / \mathrm{Cip} 1}$ and $\mathrm{p} 27^{\mathrm{Kip} 1}$ but not p53, was increased in fascin-suppressed cells during oxidative stress (Fig. 3D). In addition, cell cycle analyses indicated that fascin suppression induced G1 arrest and a concomitant decrease in the $\mathrm{S}$-phase fractions in response to mild oxidative stress that did not induce apoptotic cell death (G1-G0, 54.9\%; $\mathrm{S}, 25.4 \%$; and G2-M, $19.7 \%$ in the control cells; G1-G0, 75.1\%; S, 7.1\%; and G2-M, $17.8 \%$ fascin-suppressed cells), suggesting that fascin-induced cell senescence was involved in G1/Sphase transition and may sensitize cells to specific cell-cycle checkpoints. Withdrawal of the oxidative stress did not release the cells from growth arrest or premature senescence. The results provided support for the possibility that fascin signaling is associated with stress-induced premature senescence.

Suppression of fascin promotes apoptosis and inhibits anchorage-independent growth. To examine the effect of the altered fascin expression on apoptosis, MCF-7 cells were incubated with various concentrations of $\mathrm{H}_{2} \mathrm{O}_{2}$ for $24 \mathrm{~h}$. The TUNEL assays consistently revealed that treatment with atRA enhanced the sensitivity of cells to $\mathrm{H}_{2} \mathrm{O}_{2}$-induced cell death, whereas knocking down fascin expression significantly increased cell sensitivity to apoptosis (Fig. 4A). Fascin suppression also 
A
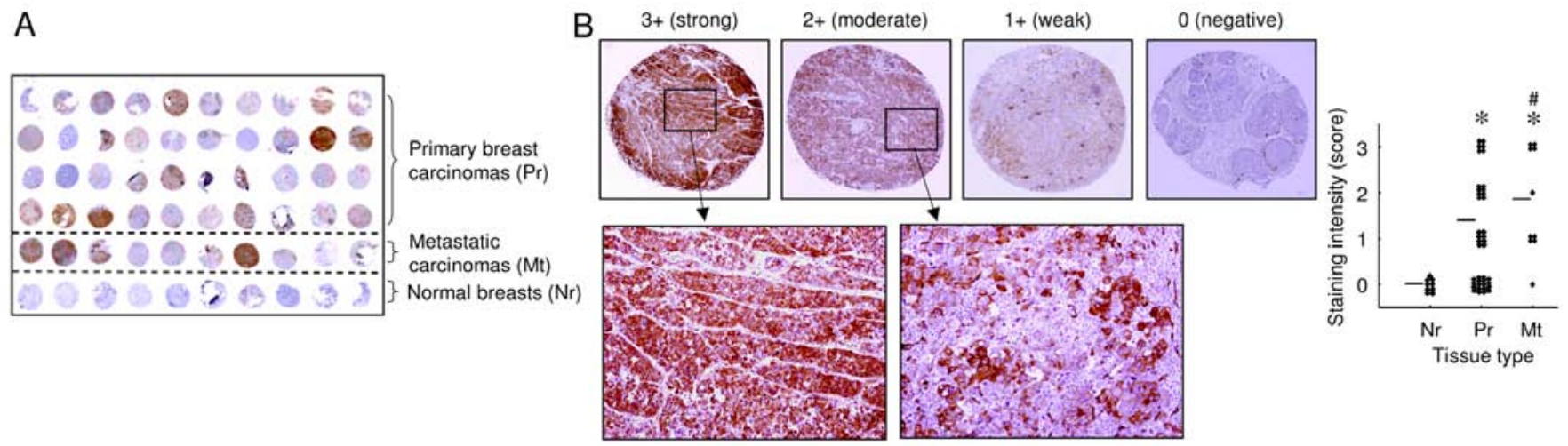

C

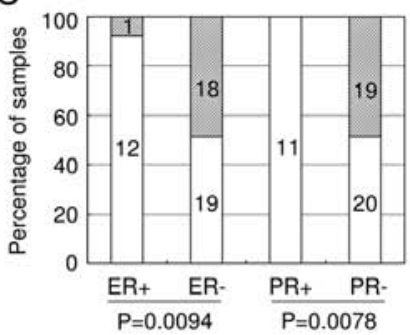

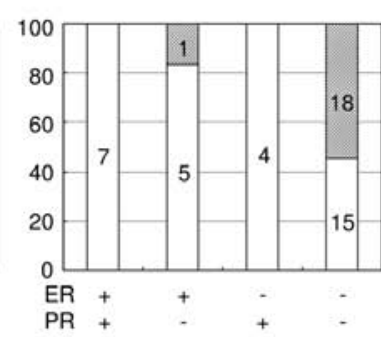

; Fascin negative $\quad P<0.0001$ ; Fascin positive
D

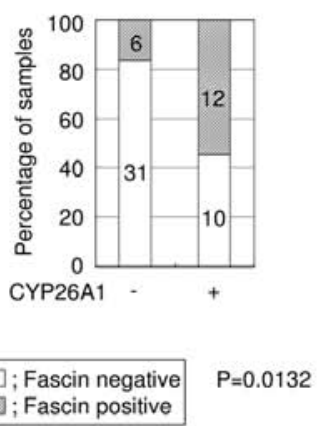

$\mathrm{E}$

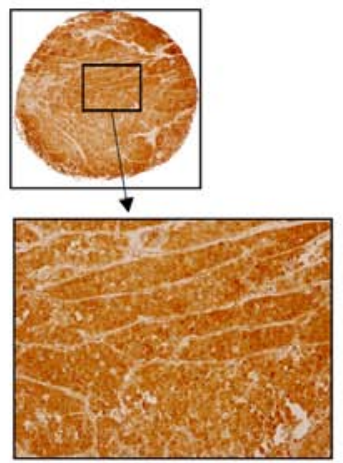

Figure 2. Increased expression of fascin in breast cancers. (A) Tissue microarray analysis shows that fascin is overexpressed in primary breast carcinomas (Pr) and metastatic carcinomas (Mt), but not in normal breast tissues (Nr). (B) Correlation between the types of tissue and the staining intensity of the representative samples (horizontal bar, mean score). $\mathrm{P}<0.05$ vs. scores from $\mathrm{Nr}\left(^{*}\right)$ or $\operatorname{Pr}\left({ }^{*}\right)$. (C and D) Differential expression of fascin in ER- or PR-positive and -negative (C), or CYP26A1-positive and -negative (D) breast carcinomas. The numbers in the bars are the total number of cases. (E) Representative example of CYP26A1 overexpression corresponding to the strong fascin expression in (B), providing additional evidence for a relationship between CYP26A1 and fascin.

A

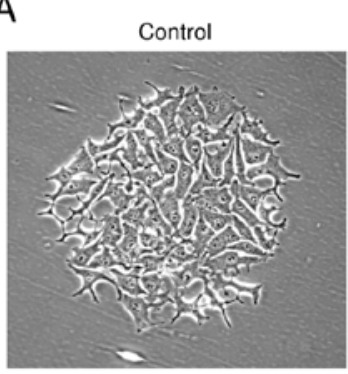

C

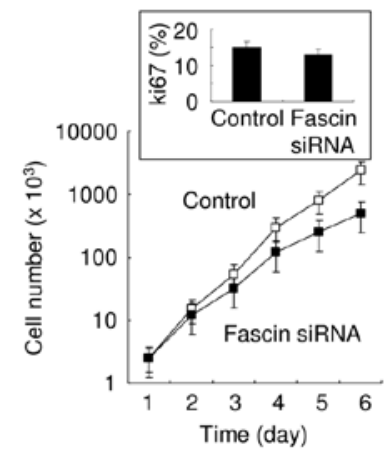

Fascin siRNA
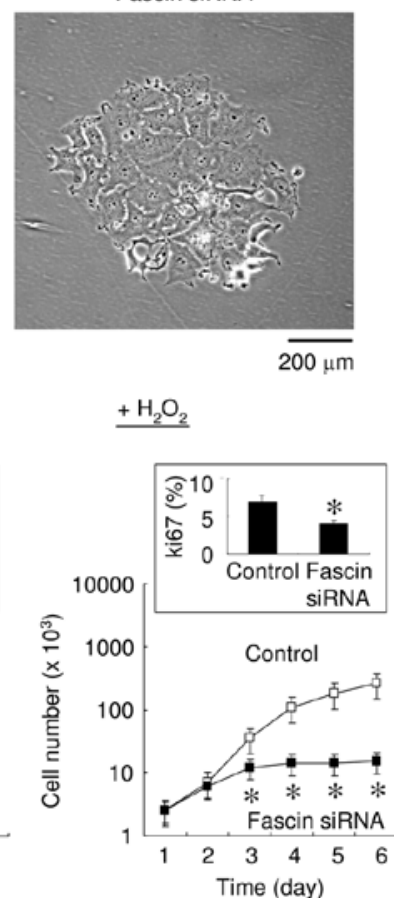

B

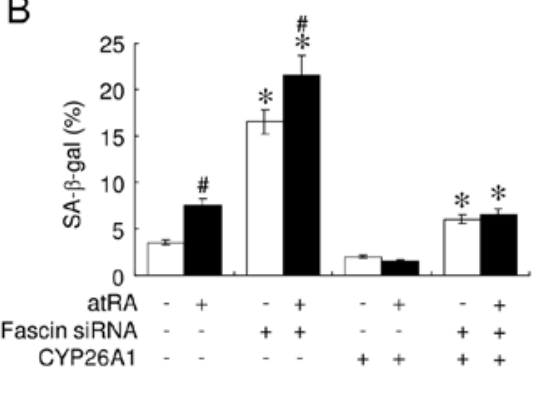

D

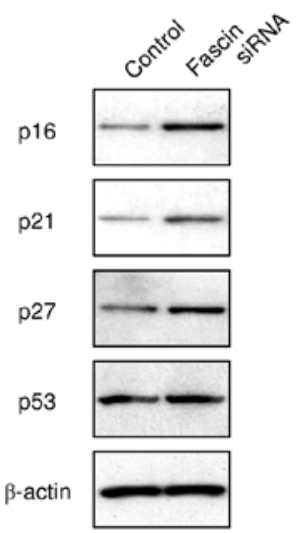

Figure 3. Suppression of fascin induces premature senescence during oxidative stress. MCF-7 cells were exposed to mild oxidative stress by treatment with $10 \mu \mathrm{M} \mathrm{H}_{2} \mathrm{O}_{2}$ for (A, B and D) 6 days or (C) up to 6 days in the presence or absence of $1 \mu \mathrm{M}$ atRA in cells transfected with fascin-specific or negative control siRNA. (A) Fascin downregulation induces a cell morphology with extended cytoplasm. (B) Quantification of SA- $\beta$-gal-stained cells. (C) Proliferation assay in cells with (right panel) and without (left panel) $\mathrm{H}_{2} \mathrm{O}_{2}$ treatment; insets show Ki-67 labeling indices on day 6 . (D) Western blot analysis of cell cycle inhibitor expression in the presence of $10 \mu \mathrm{M} \mathrm{H}_{2} \mathrm{O}_{2}$ for 6 days in cells transfected with fascin-specific or negative control siRNA. P<0.05 control cells vs. those treated with atRA $\left({ }^{\#}\right)$, and $\mathrm{P}<0.05$ control cells vs. those transfected with fascin-specific siRNA $\left(^{*}\right)$. 
A

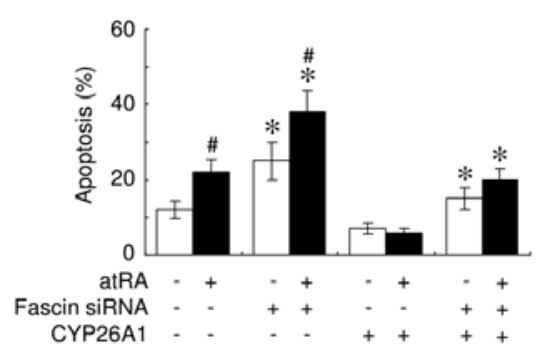

B

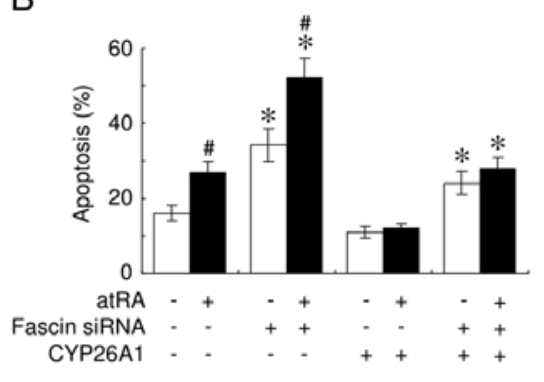

C

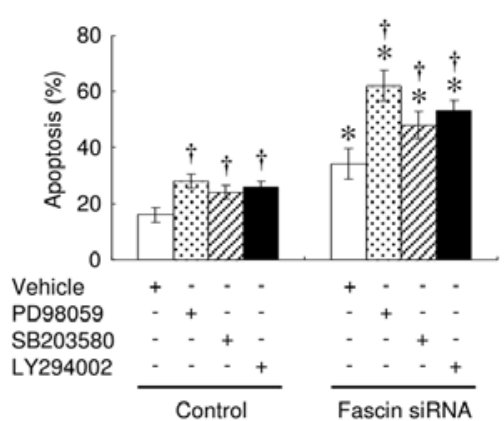

Figure 4. Suppression of fascin promotes apoptosis. (A and B) Apoptotic cell death in MCF-7 cells, as quantified using TUNEL assays. (A) Apoptosis was examined after $24 \mathrm{~h}$ of exposure to $50 \mu \mathrm{M} \mathrm{H}_{2} \mathrm{O}_{2}$ and (B) anoikis was induced, in the presence or absence of $1 \mu \mathrm{M}$ atRA in cells transfected with fascin-specific or negative control siRNA. (C) Effects of three different kinase inhibitors on anoikis. $\mathrm{P}<0.05$ control cells vs. those treated with atRA $\left({ }^{*}\right)$ or protein kinase inhibitor (') and $\mathrm{P}<0.05$ control cells vs. those transfected with fascin-specific siRNA ( ${ }^{*}$ ).

A
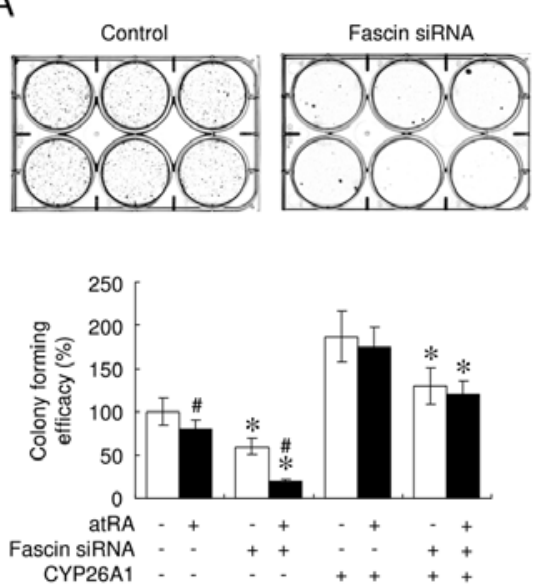
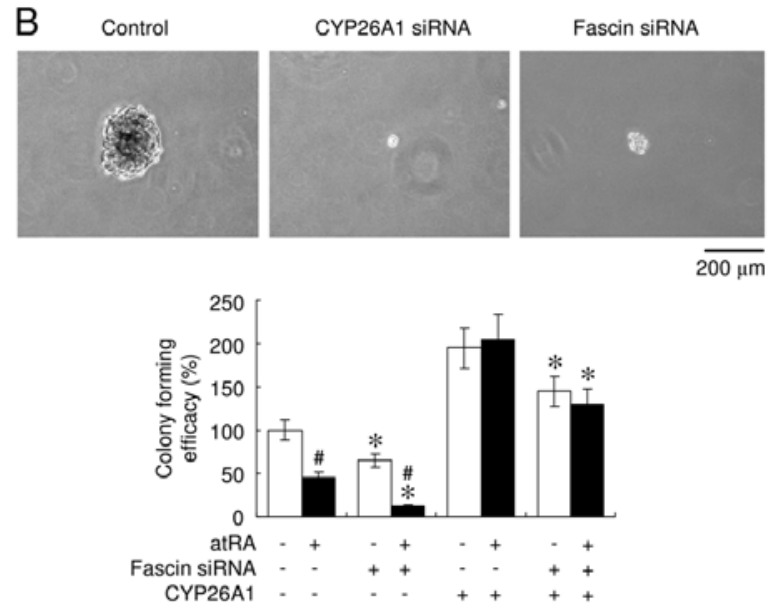

C

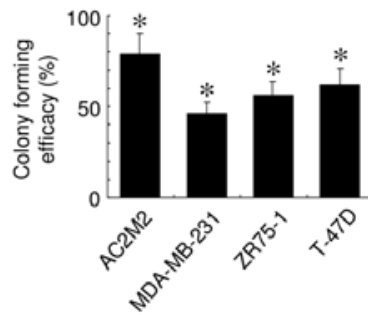

Figure 5. Suppression of fascin inhibits colony-formation. Knocking down fascin inhibits colony formation in (A) 2-D or (B and C) 3-D cultures of (A and B) MCF-7 and (C, as indicated) other cell types. (B) Similarly, knocking down of constitutive expression of CYP26A1 suppresses colony formation in $3-\mathrm{D}$ cultures of MCF-7 cells. The number of colonies formed from the control cells was defined as $100 \%$. Representative results of the Giemsa staining of cell culture plates to assess 2-D (A, upper panel) and 3-D (B, upper panel) colonies, and quantitative analyses of colony formation (A and B, lower panel, and C). $\mathrm{P}<0.05$ control cells vs. those treated with atRA $\left(^{*}\right)$, and $\mathrm{P}<0.05$ control cells vs. those transfected with fascin-specific siRNA $\left(^{*}\right)$. atRA, all-trans retinoic acid.

promoted atRA-mediated enhanced anoikis (Fig. 4B). Anoikis was also observed in the event of fascin suppression, even when the cells overexpressed CYP26A1. However, atRA had no effect on anoikis in CYP26A1-overexpressing cells.

To investigate the signal transduction pathways involved in the effects of fascin on apoptotic sensitivity, various kinase inhibitors were used (Fig. 4C). The PD98059 MAPK inhibitor efficiently induced anoikis, which was increased further when the fascin expression was suppressed. The p38 MAPK inhibitor SB203580 and the PI3K inhibitor LY294002 had significant, but much less pronounced, effects compared to PD98059.

Suppressing fascin expression also significantly inhibited colony formation, a measure of cell transformation, by decreasing the number and size of colonies in 2-D and 3-D cultures (Fig. 5A and B). In addition, downregulation of the CYP26A1 expression significantly suppressed colony formation in soft agar (Fig. 5B), supporting the presence of a direct mechanistic link between CYP26A1 expression and oncogenic behavior. Treatment with atRA consistently inhibited anchorage-independent growth and enhanced the effects of fascin downregulation. These observations suggested that atRA and fascin suppression synergistically inhibit anchorage-independent growth properties. The knockdown of fascin expression inhibited the efficacy of colony formation in the CYP26A1-overexpressing MCF-7 cells. Similarly, fascin suppression significantly inhibited colony formation in various types of breast carcinoma cells (Fig. 5C). These results suggested that fascin expression modulates colony formation in CYP26A1-expressing cells.

Suppression of fascin decreases cell invasion and motility. To determine whether fascin modulated cell invasion, an invasion assay with Matrigel ${ }^{\mathrm{TM}}$ was employed using MDA-MB-231 cells, since the MCF-7 cells were not sufficiently invasive. Suppression of the constitutive expression of CYP26A1 in the MDA-MB-231 cells significantly reversed the CYP26A1mediated effects, including invasiveness (Fig. 6A). When fascin was knocked down using siRNA, a significantly decreased invasion was observed and treatment with atRA potentiated these effects (Fig. 6A and B). Fascin suppression partially, 
A
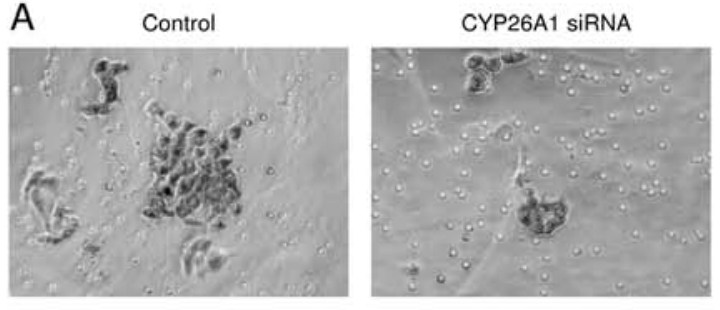

D
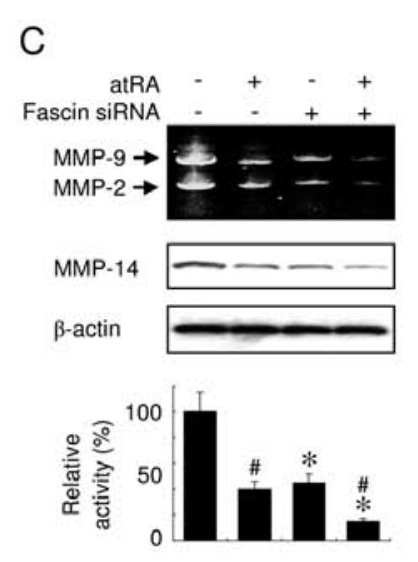
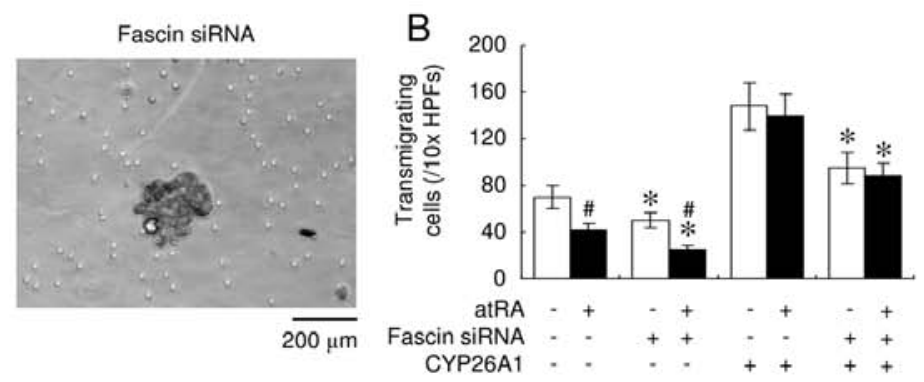
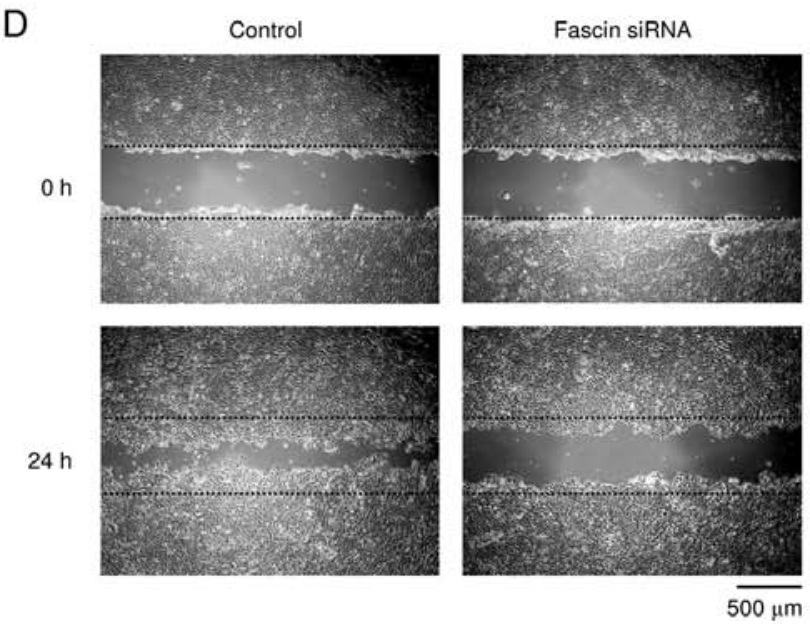

Figure 6. Suppression of fascin decreases cell invasion and motility. MDA-MB-231 cells transfected with fascin-specific or negative control siRNA were assayed in the presence or absence of $1 \mu \mathrm{M}$ atRA. (A and B) Knockdown of fascin or CYP26A1 suppresses cell invasion. (A) Representative results of an invasion assay and (B) quantitative analysis of the invading cells. (C) Gelatin zymography was used to determine MMPs (MMP-2 and -9) activity (upper panel) and quantification of active MMP-9 (lower panel) in a zymogram. The production of active MMP-14 was also analyzed by western blotting. (D) Cell migration in monolayer wound healing assays. Images were captured $24 \mathrm{~h}$ after standard wound scraping. The dotted lines denote the edge of the initial wound. $\mathrm{P}<0.05$ control cells vs. those treated with atRA $\left({ }^{*}\right)$, and $\mathrm{P}<0.05$ control cells vs. those transfected with fascin-specific siRNA $\left({ }^{*}\right)$. atRA, all-trans retinoic acid.

but significantly decreased the invasiveness of the CYP26A1overexpressing cells, whereas atRA had no effect. Gelatin zymography was then performed to assess MMP-2 and -9 . The results of the assay revealed decreases in the activities of the two MMPs when fascin was downregulated (Fig. 6C). In addition, fascin suppression resulted in the decreased expression of MMP-14, which is known to be a pro-invasive marker, and enhanced the atRA-mediated downregulation of MMP-14. The monolayer wound-healing assay clearly demonstrated that decreased fascin expression significantly inhibited cell migration (Fig. 6D). These observations indicated that fascin suppression inhibited the cell invasion ability and motility of the CYP26A1-expressing cells and suggest that atRA has a synergistic impact on fascin via its suppressive effect.

\section{Discussion}

The expression levels of fascin increased significantly in response to the constitutive overexpression of CYP26A1 and conversely treatment with atR A downregulated the expression of fascin, suggesting that fascin expression is modulated by the intracellular RA status, regulated by the expression of CYP26A1. In addition, primary breast carcinomas, particularly hormone receptor-negative carcinomas and CYP26A1-overexpressing cancers, expressed elevated levels of fascin. Since tumors expressing ER and PR generally exhibit a less aggressive phenotype and are associated with an increased disease-free and overall survival, these data support a potential correlation between fascin overexpression and aggressive tumor behavior, and provide evidence of a correlation between CYP26A1 and fascin. Our observations also showed that siRNA-mediated suppression of fascin inhibited the malignant behavior of CYP26A1-expressing breast carcinoma cells. CYP26A1 exerts oncogenic functions during breast carcinogenesis; therefore, CYP26A1-mediated oncogenic effects may be at least partially responsible for the elevated expression of fascin. Nevertheless, it is difficult to conclude that fascin directly mediates the effect of CYP26A1. Given that suppressing fascin expression had significant, but partial effects on the CYP26A1-overexpressing cells, the possibility that the CYP26A1-mediated oncogenic effects are governed by a regulatory mechanism that is independent of fascin cannot be excluded.

The present study did not identify how intracellular RA modulates the expression of fascin. Of note, the bioinformatics analyses did not identify a putative RA response element in the fascin promoter, suggesting that atRA does not regulate fascin expression directly. One possible explanation for this is that atRA alters the activity of other trans-acting nuclear transcription factors and/or co-activators. The fascin promoter contains a number of binding motifs including those for p300 and CREB-binding protein (CBP). Previous findings have demonstrated that the atRA-mediated differentiation of F9 embryonic carcinoma cells was inhibited by a ribozyme 
directed against $p 300 \mathrm{mRNA}(18)$. In addition, fascin expression was significantly decreased in CBP-depleted NT2 neuronal cells (19). However, direct comparisons among cell lines are challenging since various cell types may exhibit unique activation pathways that may be mutated or dysregulated. These changes may provide erroneous data with regard to the sensitivity of cells to atRA. On the other hand, the present study has clearly demonstrated that the CYP26A1-mediated increase in fascin expression occurs at the transcriptional level. Nevertheless, this does not exclude the possibility that fascin is also regulated by post-transcriptional mechanisms, although the post-transcriptional regulation of fascin has not been reported.

CYP26A1- and fascin-mediated cell events may be mediated via the MAPK signaling pathway (10). Such crosstalk between CYP26A1 and fascin signaling suggests that CYP26A1 indirectly regulates fascin expression. Given the complexity of the cell signaling events that regulate epithelial cell apoptosis, proliferation and differentiation, it is likely that CYP26A1 and fascin modulate a variety of signal transduction molecules that regulate tumor pathology. This is partially supported by the CYP26A1 overexpression-mediated changes in gene-expression, suggesting that a number of genes that favor cell survival are modulated by CYP26A1.

Non-cytotoxic cell stress induces premature senescence, which is characterized by the upregulation of negative cell cycle regulators (20). The present findings have shown that fascin suppression induced premature senescence, which was accompanied by the upregulation of $\mathrm{p} 16^{\mathrm{INK} 4 \mathrm{~A}}, \mathrm{p} 21^{\text {Waf1/Cip1 }}$ and $\mathrm{p} 27^{\mathrm{Kip} 1}$. Although CDK inhibitors are the key mediators of growth arrest during premature senescence, whether the upregulation of CDK inhibitors directly contributes to the senescence-associated phenotype in fascin-specific siRNA-expressing cells remains unclear. However, it is possible that the downregulation of fascin provokes premature senescence via the enhanced expression of CDK inhibitors. By contrast, no changes in p53 were detected during premature senescence, suggesting that the senescence associated with fascin signaling was mediated by $\mathrm{p} 53$-independent mechanisms and/or mutant $\mathrm{p} 53$. The functional requirements for CDK inhibitors in the senescent state appear to be heterogeneous under each experimental condition (20). For example, a p53-independent senescence mechanism was suggested to be responsible for hypermitogenic proto-oncogenic ERBB2 signaling in breast carcinoma cells (21). Another study reported SA- $\beta$-gal positivity in clinical breast cancer cells, suggesting that chemotherapy-induced DNA damage induced senescence in vivo and that SA- $\beta$-gal staining was associated with low levels of p53 (22).

The present study provides evidence that fascin signaling may be associated with premature senescence and apoptosis. It is well accepted that senescence involves aspects of the apoptotic machinery, and is an antioncogenic fail-safe mechanism that may function as a natural brake to tumor development (23). Previously, we suggested that strategies to decrease CYP26A1 activity may be a significant method to increase sensitivity of cancer cells to pro-apoptotic agents (10). By contrast, a number of reports have successfully demonstrated that DNA-damaging agents induce cell senescence, even in cells with reduced apoptotic responsiveness (24). Therefore, the induction of fascin signaling-associated cell senescence may have potential as an anticancer mechanism to inhibit oncogenic activity by inducing cancer cells to exit uncontrolled proliferation. Given the similarities between the apoptotic and senescence machineries, the CYP26A1-fascin axis may be an appropriate therapeutic target for breast cancer.

\section{Acknowledgements}

The present study was supported in part by grants from the Grants-in-Aid for Scientific Research from the Japan Society for the Promotion of Science and the Smoking Research Foundation of Japan.

\section{References}

1. Adams JC: Roles of fascin in cell adhesion and motility. Curr Opin Cell Biol 16: 590-596, 2004.

2. Kureishy N, Sapountzi V, Prag S, Anilkumar N and Adams JC: Fascins, and their roles in cell structure and function. Bioessays 24: 350-361, 2002.

3. Edwards RA and Bryan J: Fascins, a family of actin bundling proteins. Cell Motil Cytoskeleton 32: 1-9, 1995.

4. Grothey A, Hashizume R, Ji H, Tubb BE, Patrick CW Jr, Yu D, Mooney EE and McCrea PD: C-erbB-2/ HER-2 upregulates fascin, an actin-bundling protein associated with cell motility, in human breast cancer cell lines. Oncogene 19: 4864-4875, 2000.

5. Grothey A, Hashizume R, Sahin AA and McCrea PD: Fascin, an actin-bundling protein associated with cell motility, is upregulated in hormone receptor negative breast cancer. Br J Cancer 83: $870-873,2000$

6. Jawhari AU, Buda A, Jenkins M, Shehzad K, Sarraf C, Noda M, Farthing MJ, Pignatelli $\mathrm{M}$ and Adams JC: Fascin, an actinbundling protein, modulates colonic epithelial cell invasiveness and differentiation in vitro. Am J Pathol 162: 69-80, 2003.

7. Hashimoto Y, Ito T, Inoue H, Okumura T, Tanaka E, Tsunoda S, Higashiyama M, Watanabe G, Imamura $M$ and Shimada $Y$ : Prognostic significance of fascin overexpression in human esophageal squamous cell carcinoma. Clin Cancer Res 11: 2597-2605, 2005.

8. Hwang JH, Smith CA, Salhia B and Rutka JT: The role of fascin in the migration and invasiveness of malignant glioma cells. Neoplasia 10: 149-159, 2008.

9. Hayashi Y, Osanai M and Lee GH: Fascin-1 expression correlates with repression of E-cadherin expression in hepatocellular carcinoma cells and augments their invasiveness in combination with matrix metalloproteinases. Cancer Sci 102: 1228-1235, 2011.

10. Osanai M, Sawada N and Lee GH: Oncogenic and cell survival properties of the retinoic acid metabolizing enzyme, CYP26A1. Oncogene 29: 1135-1144, 2010.

11. Chang CL, Hong E, Lao-Sirieix P and Fitzgerald RC: A novel role for the retinoic acid-catabolizing enzyme CYP26A1 in Barrett's associated adenocarcinoma. Oncogene 27: 2951-2960, 2008.

12. Shelton DN, Sandoval IT, Eisinger A, Chidester S, Ratnayake A, Ireland CM and Jones DA: Up-regulation of CYP26A1 in adenomatous polyposis coli-deficient vertebrates via a WNT-dependent mechanism: Implications for intestinal cell differentiation and colon tumor development. Cancer Res 66: 7571-7577, 2006.

13. Brown GT, Cash BG, Blihoghe D, Johansson P, Alnabulsi A and Murray GI: The expression and prognostic significance of retinoic acid metabolising enzymes in colorectal cancer. PLoS One 9: e90776, 2014.

14. Elliott BE, Maxwell L, Arnold M, Wei WZ and Miller FR: Expression of epithelial-like markers and class I major histocompatibility antigens by a murine carcinoma growing in the mammary gland and in metastases: Orthotopic site effects. Cancer Res 48: 7237-7245, 1988 .

15. Dimri GP, Lee X, Basile G, Acosta M, Scott G, Roskelley C, Medrano EE, Linskens M, Rubelj I, Pereira-Smith O, et al: A biomarker that identifies senescent human cells in culture and in aging skin in vivo. Proc Natl Acad Sci USA 92: 9363-9367, 1995.

16. Murray GI, Patimalla S, Stewart KN, Miller ID and Heys SD: Profiling the expression of cytochrome P450 in breast cancer. Histopathology 57: 202-211, 2010.

17. Ben-Porath I and Weinberg RA: When cells get stressed: An integrative view of cellular senescence. J Clin Invest 113: 8-13, 2004. 
18. Kawasaki H, Eckner R, Yao TP, Taira K, Chiu R, Livingston DM and Yokoyama KK: Distinct roles of the co-activators p300 and CBP in retinoic-acid-induced F9-cell differentiation. Nature 393: 284-289, 1998

19. Megiorni F, Indovina P, Mora B and Mazzilli MC: Minor expression of fascin-1 gene (FSCN1) in NTera 2 cells depleted of CREB-binding protein. Neurosci Lett 381: 169-174, 2005.

20. McConnell BB, Starborg M, Brookes S and Peters G: Inhibitors of cyclin-dependent kinases induce features of replicative senescence in early passage human diploid fibroblasts. Curr Biol 8: 351-354, 1998

21. Trost TM, Lausch EU, Fees SA, Schmitt S, Enklaar T, Reutzel D, Brixel LR, Schmidtke P, Maringer M, Schiffer IB, et al: Premature senescence is a primary fail-safe mechanism of ERBB2-driven tumorigenesis in breast carcinoma cells. Cancer Res 65: 840-849, 2005.
22. te Poele RH, Okorokov AL, Jardine L, Cummings J and Joel SP: DNA damage is able to induce senescence in tumor cells in vitro and in vivo. Cancer Res 62: 1876-1883, 2002.

23. Sherr CJ and DePinho RA: Cellular senescence: Mitotic clock or culture shock? Cell 102: 407-410, 2000.

24. Chang BD, Broude EV, Dokmanovic M, Zhu H, Ruth A, Xuan Y, Kandel ES, Lausch E, Christov K and Roninson IB: A senescence-like phenotype distinguishes tumor cells that undergo terminal proliferation arrest after exposure to anticancer agents. Cancer Res 59: 3761-3767, 1999. 